$\$$ Research Square

\title{
A Lightweight Neural Network Model For Battery State Of Health
}

\section{Danhua Zhou ( $\nabla$ DanHZhou@163.com )}

Jiangsu Academy of Safety Science And Technology https://orcid.org/0000-0002-8808-9956

\section{Research Article}

Keywords: Lithium-ion battery, State of health, Temporal convolutional network

Posted Date: February 2nd, 2022

DOl: https://doi.org/10.21203/rs.3.rs-994980/v1

License: (9) This work is licensed under a Creative Commons Attribution 4.0 International License. Read Full License 


\title{
A Lightweight Neural Network Model For Battery State Of Health
}

\section{Estimation}

Danhua Zhou

Jiangsu Academy Of Safety Science And Technology, Nanjing 210000 China

Corresponding author: Danhua Zhou (DanHZhou@163.com).

\section{HIGHLIGHTS}

- Feature extraction is automatic and independent of global charging data to improve model reliability.

- Convolutional attention mechanism is used to enhance or suppress detailed features.

- Depthwise separable convolution is used to extend TCN model to multivariate prediction model.

\begin{abstract}
Accurate estimation of the state of health ( $\mathrm{SOH}$ ) of lithium-ion batteries is the key to ensure the safe use of lithium-ion batteries. In practice, the application of traditional health features is hindered by incomplete charge and discharge. When the battery is stably charged, the voltage and temperature of the battery under different health states show similar spatial degradation trends. Therefore, the degradation trend of voltage and temperature is directly taken as the health characteristic sequence through the dynamic time warping barycenter averaging (DBA) clustering. In addition, a new model attention depthwise temporal convolutional network (AD-TCN) considering health characteristics is proposed for $\mathrm{SOH}$ estimation. Depthwise separable convolution operation is used to extend temporal convolutional network (TCN) to a model suitable for multivariate prediction. Depthwise convolution is used as feature extractor, and pointwise convolution recombines all features for regression prediction. In addition, the convolutional block attention module is used in the channel dimension and spatial dimension to selectively enhance or suppress the details. Experiments on NASA data sets show that this method has strong reliability and high prediction accuracy.
\end{abstract}

Keywords:

Lithium-ion battery

State of health

Temporal convolutional network 


\section{Introduction}

Lithium-ion batteries have been widely applied to human life because of its fast charging speed, low self-discharge, long service life and so on. Now the application of lithium-ion batteries has expanded from portable electronic devices (such as mobile phones, cameras, and laptops) to larger applications (such as new energy vehicles, drones and satellites), and other larger industrial equipment and electrical energy storage are inseparable from it [1]. Lithium-ion battery is generally recognized as a complex electrochemical system, except that the environmental impact, the performance itself there are many uncertainties. Precisely because of this, how to accurately predict the state of health ( $\mathrm{SOH}$ ) of lithium-ion batteries is the key to ensuring their safe and reliable use.

In the process of recycling, the total capacity of lithium-ion batteries will decrease and the internal resistance will increase. This phenomenon indicates that batteries have the characteristic of declining energy storage capacity, which is referred to as cell aging [2]. $\mathrm{SOH}$ is a standard for evaluating the aging degree of batteries, which essentially reflects the aging and damage degree of lithium-ion batteries. Battery aging is a process that the decline of itself various functions, which is a complex nonlinear process, and the factors involved are awfully wide [3]. In general, when the actual capacity of cell drops to $70 \%-80 \%$ of the standard capacity, the service life of battery will come to end. If the battery continues to be used at this time, the operation performance of the entire system may be affected, even resulting in disasters. Therefore, it is momentous to design a method to accurately and efficiently monitor $\mathrm{SOH}$ to ensure that battery is replaced before it fails.

At present, the method of $\mathrm{SOH}$ estimation can be divided into two categories, the first category is the mathematical model method that needs to consider the internal chemical mechanism of the lithium-ion battery, and the second category is the data-driven method that indirectly predicts based on the measurement data. Model-based method mainly include electrochemical model [4][5] and equivalent circuit model [6][7]. The electrochemical model mainly calculates the accurate $\mathrm{SOH}$ by studying the internal electrochemical reaction of the battery. However, the modeling of $\mathrm{SOH}$ based on electrochemical model is complex and difficult to be applied in practice. The equivalent circuit model achieves the effect of simulating battery characteristics through the circuit system composed of electronic components. Although this method is less difficult than the electrochemical model, the essence of the equivalent circuit is approximate processing, and the deviation of any parameter may lead to a large SOH calculation error. In addition, the biggest problem to be solved with model-based approach is that currently there is no suitable model that can accurately simulate all the aging behavior [8]. [9] mentioned in 2020 the application of deep learning in the prognostics and health management field, which deserves our attention.

With the development of the big data, more data-driven methods have shown their respective advantages in $\mathrm{SOH}$ estimation. Data-driven $\mathrm{SOH}$ estimation can be divided into two categories. The first category defines $\mathrm{SOH}$ estimation and remaining useful life (RUL) prediction as time series processing problem, and only considers the capacity series as input to the model. This kind of method takes the local capacity regeneration in lithium-ion batteries into account. This phenomenon is explained by the side reaction between the electrolyte and the electrodes during the charging and discharging of the lithium-ion battery, which leads to a decline in electrochemical performance, but when the 
battery is not working, the electrochemical performance will be restored to a certain extent, so in the process of overall capacity decay, there will be local peaks that change the overall attenuation trend. At present, most of the methods for accurately capturing this phenomenon rely on the combination technology. The wavelet decomposition or empirical mode decomposition is used to decompose the capacity sequence, and then use the commonly used single models (e.g. Gated Recursive Unit (GRU) and Autoregressive model) or combined model (e.g. Long Short Term Memory (LSTM) combined with Elman [10], extreme value learning machine combined particle filter [11] and logistic regression combined Gaussian process regression [12][13] etc.). This method has the advantage of high prediction accuracy, and the local regeneration phenomenon of the capacity sequence can be accurately captured[14].

However, the shortcomings of the first method are also obvious. It only considers the capacity sequence and does not consider other factors besides capacity (such as voltage, current, and temperature), so the accuracy of the actual online estimation of lithium-ion batteries will be affected. The second category is the most researched method that considers health features (HFs) in current data-driven methods[15]. The research and improvement of this kind of method mainly focus on how to extract features with high correlation and how to improve the prediction accuracy and efficiency of the model. In terms of HFs extraction, [16] proposes using the sample entropy of the discharge voltage as HFs. HFs used by [17] is the isobaric time difference of the discharge curve. [18] based on the duration of the constant current $(\mathrm{CC})$ mode, the duration of the constant voltage $(\mathrm{CV})$ model, the slope of the curve at the end of the $\mathrm{CC}$ charging model and the vertical slope of the $\mathrm{CC}$ charging curve angle as HFs realized $\mathrm{SOH}$ monitoring with high precision.[19] summarize the recent extraction methods based on HFs. Fourteen features are extracted from charging voltage, current and temperature, and 9 features with high correlation degree are selected by Principal Component Analysis (PCA) dimensionality reduction. In addition, many recent paper focus the feature extraction on incremental capacity analysis [20][21]. However, although there are many methods for extracting features, it is up to the prediction model itself to improve the prediction accuracy and stability. In terms of model prediction, Particle Filtering (PF)[22], Support Vector Machine (SVM) [23] and Relevance Vector Machine (RVM) [24] were used long before to predict battery aging. In recent years, more researches are on improved Gaussian process regression algorithms [25][26][27] and Recurrent Neural Network (RNN) [28][29][30][31][32] to achieve better prediction of $\mathrm{SOH}$ effect.

Based on the above research, considering the $\mathrm{SOH}$ estimation method of HFs, the following points need to be improved. In terms of feature extraction, it must be admitted that selecting a good angle to extract highly relevant features is important for $\mathrm{SOH}$ estimation. In addition, according to the research in the literature [19], it can be found that the correlation degree obtained by different feature extraction methods for cells under different working conditions are uneven. Thus, a feature extraction method with high accuracy applicable to all batteries is currently not available, and some feature extraction methods still have the limitation of relying on the entire charge and discharge process. Secondly, many existing studies only analyze the importance of features from the perspective of correlation degree, but do not improve the effect of high correlation degree features on the prediction results from the model essence. Besides, the SOH estimation problem of HFs is essentially 
a multi-sequence prediction problem. Currently, the models that can be improved for $\mathrm{SOH}$ estimation are mostly concentrated on traditional algorithms or models that have proven to perform well in the field of battery performance. In recent years, deep learning models have benefited from the development of data. There are many models that excel in sequence prediction and should be considered for application and improvement.

In view of the above problems, the contributions of this paper are mainly divided into the following points:

(1) The degradation trend series of voltage and temperature response during charge and discharge is directly clustered through DTW (dynamic time warping) barycenter averaging (DBA) algorithm as the input of the model. The convolutional neural network has the performance of extracting features through multiple convolution operations, and the features extracted by deep convolution have been proven to be better than the manually extracted features in many places [33].

(2) The depthwise separable convolution operation is used to expand the Temporal Convolutional Network (TCN) into a multiple sequence prediction model that can consider HFs. Maintain the superior performance of TCN on single sequence prediction problems.

(3) Combined with Convolutional Block Attention Module (CBAM), a new ADTCN model is proposed.

In the actual use scenario, the charging and discharging of the battery depends on the user's mode, so these processes are random. Many commonly used fixed points and angles as the input features of the model are not applicable to all occasions, so such methods have certain limitations. This study considers that the battery capacity degradation trend is similar regardless of the charging and discharging methods and the environment, so this study improves the reliability of the method by using part of the degradation trend as feature input. Secondly, the focus of this study is to propose a new AD-TCN model for multivariate $\mathrm{SOH}$ estimation. To a certain extent, the characteristics of data determine the upper limit of prediction accuracy, and the key to whether this upper limit can be approached indefinitely lies in the prediction model itself.

The structure of this paper is as follows: First, the batteries in the NASA battery database are analyzed in section 2 , and the degradation trend sequence of the lithium-ion battery is preprocessed as the input of the model. Section 3 presents the model structure for $\mathrm{SOH}$ estimation. Then, the $\mathrm{SOH}$ estimation results are analyzed in section 4 , and finally discussed in section 5 .

\section{Data Preprocessing}

In this study, a total of four recycled battery data were used. First, the specifics of the data used were introduced in part 2.1, then the capacity degradation sequence was analyzed in part 2.2, and finally part 2.3 clustered the sequence through the DBA algorithm as the final input feature.

\subsection{Data analysis of cell aging experiment}

The public lithium-ion battery dataset used in this study comes from the NASA Ames Prognostics Center of Excellence (PCOE) [34]. The experiment used commercial 18650 lithium-ion battery with rated capacity of 2.0 $\mathrm{Ah}$, and a cyclic charge and discharge test sampling at room temperature of $24^{\circ} \mathrm{C}$. In this study, the battery data of the battery numbers B0005, B0006, B0007 and B0018 were selected for analysis. The charging data was collected using a constant current and constant voltage (CC-CV) method. In the $1.5 \mathrm{~A}$ constant current charging mode until the voltage reached $4.2 \mathrm{~V}$, then charge at constant voltage 
until the current drops to $20 \mathrm{~mA}$. The discharge condition is constant current $2 \mathrm{~A}$ discharge until the voltage drops to $2.7 \mathrm{~V}, 2.5 \mathrm{~V}, 2.2 \mathrm{~V}$ and $2.5 \mathrm{~V}$ respectively. Detailed information is given in Table 1 . The capacity curves of the four cells are given in Fig.1. From this figure, we can see that the battery capacity attenuation paths are very similar, and there is local regeneration of the capacity, which requires that the model used for $\mathrm{SOH}$ estimation has good stability and reliability.

Table 1. Test lithium-ion batteries information

\begin{tabular}{cccccc}
\hline \hline Battery ID & Discharge current & Rated capacity & $\mathrm{CC} / \mathrm{CV}$ & End voltage & Temperature $\left({ }^{\circ} \mathrm{C}\right)$ \\
\hline B0005 & $2 \mathrm{~A}$ & $2 \mathrm{Ah}$ & $1.5 \mathrm{~A} / 20 \mathrm{~mA}$ & $2.7 \mathrm{~V}$ & 24 \\
B0006 & $2 \mathrm{~A}$ & $2 \mathrm{Ah}$ & $1.5 \mathrm{~A} / 20 \mathrm{~mA}$ & $2.5 \mathrm{~V}$ & 24 \\
B0007 & $2 \mathrm{~A}$ & $2 \mathrm{Ah}$ & $1.5 \mathrm{~A} / 20 \mathrm{~mA}$ & $2.2 \mathrm{~V}$ & 24 \\
B0018 & $2 \mathrm{~A}$ & $2 \mathrm{Ah}$ & $1.5 \mathrm{~A} / 20 \mathrm{~mA}$ & $2.5 \mathrm{~V}$ & 24 \\
\hline \hline
\end{tabular}

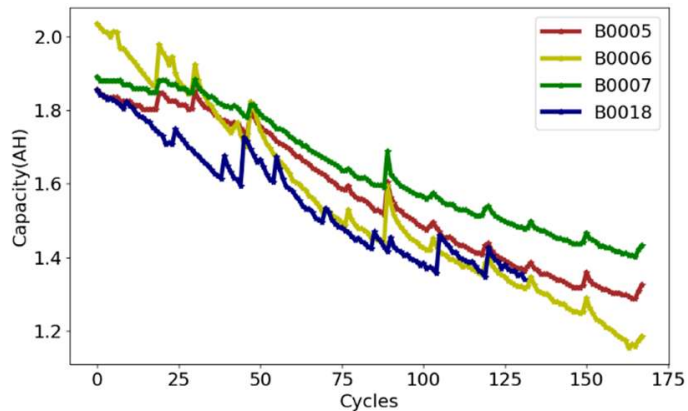

Fig.1. Capacity degradation curves of batteries

\subsection{Degenerate sequence analysis}

Considering the shortcomings of existing feature extraction methods, for different lithium-ion batteries, the correlation of the same feature extraction method on different batteries is uneven, and some feature extraction methods rely on the entire charge and discharge process. Secondly, the existing research usually extracts features from the charge-discharge voltage curve and ignores the influence of temperature. Based on the change process of voltage and temperature in the constant current charging mode, this study find that the aging trend of the battery is spatially consistent in different time periods, as shown in Fig. 2. Considering that the time of each data collection is not exactly the same, we use the data collection time point NO. to represent the time point of each data collection. It can be found from the Fig. 2 that, except for the voltage instability during the initial charging period, the voltage and temperature degradation trends in the overall constant current charging process are spatially consistent. The stable voltage and temperature in the process of degradation trend are directly used as features in this study, and simple and reliable features are used to verify the efficient performance of our model.

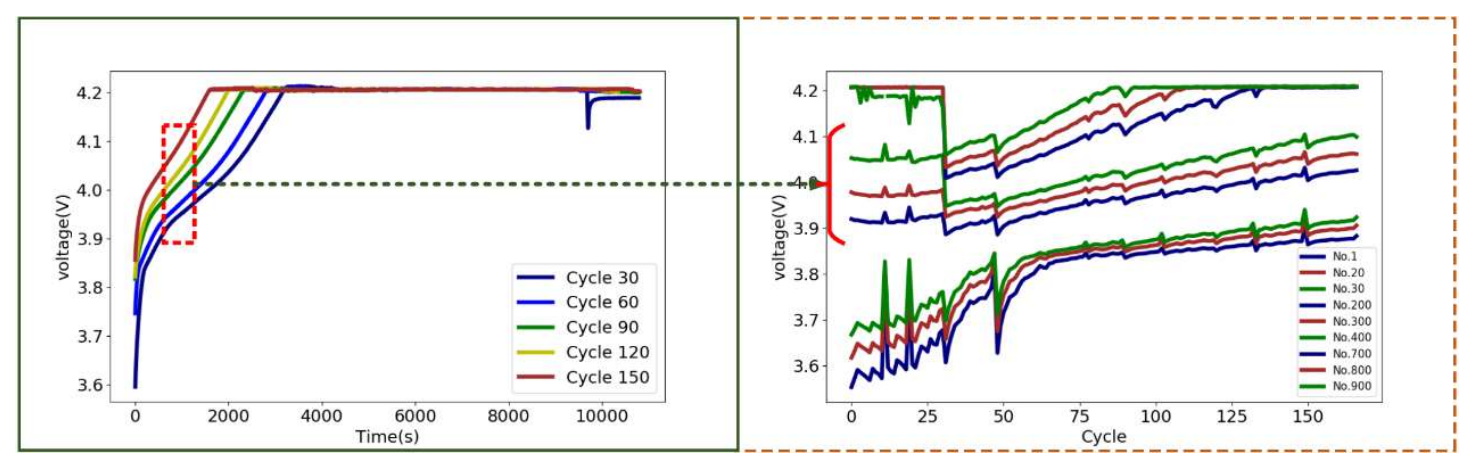




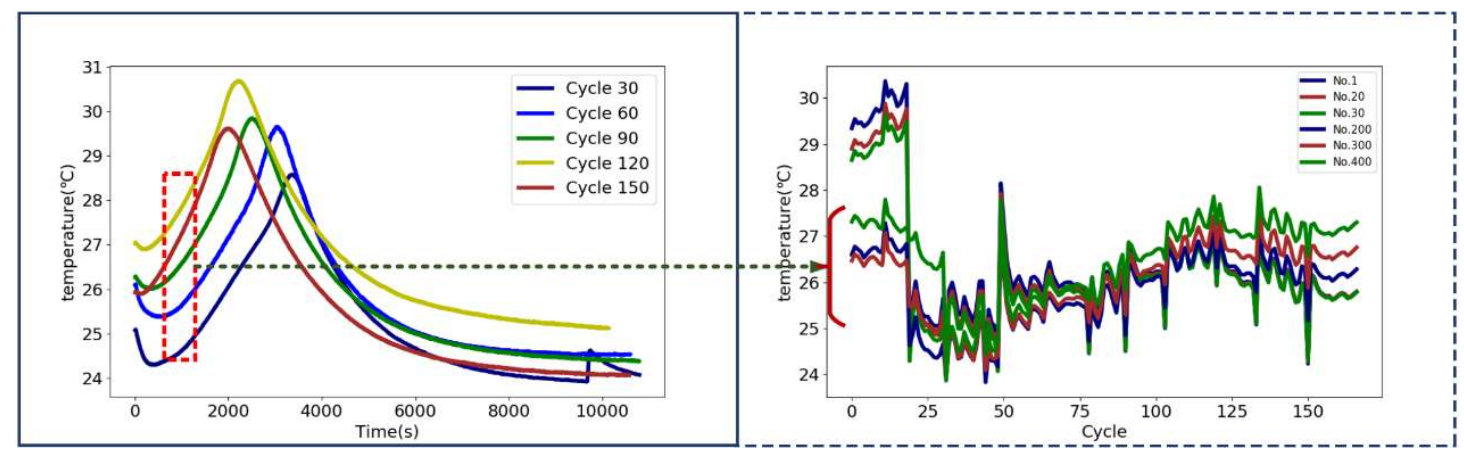

(b)

Fig. 2. (a) Battery B0005 charging voltage degradation trend curve. (b) Battery B0005 charging temperature degradation trend curve.

\subsection{DBA clustering}

With the development of deep models, automatic feature extraction through the model can reduce the impact of prediction errors due to feature extraction errors to a certain extent, and also avoid the tedious process of manually extracting features. Existing deep network methods usually input all the data directly, which will also bring a huge calculation burden to the model [25]. This study found that the cell attenuation curve has strong spatial similarity. However, it is inefficient to repeatedly extract features from similar curves. Therefore, this paper using DBA algorithm proposes to cluster the voltage and temperature degradation sequences to reduce the effect of repeated feature extraction. DBA algorithm is proposed in [35]. In deep learning, DBA algorithm is commonly used to cluster time series or enhance data. Since the characteristics of the time series may be misaligned on the time axis, the method of averaging each point in the time series set to calculate the average time series in a one-to-one correspondence is prone to abnormal values. The DBA algorithm uses the size of the dynamic time warping (DTW) distance [36] as the optimization index for obtaining the average sequence, thus achieving good results in the problem of calculating the average sequence.

The specific steps of the DBA algorithm are as follows:

(1) Randomly select one from the sequence set as the average sequence.

(2) Calculate the DTW distance between the current average sequence and each sequence in the sequence set, and match the coordinates of the average sequence with the coordinates of other sequences in the sequence set. The DTW distance method is calculated as follows: Suppose there are two sequence $A=\left(a_{1}, a_{2}, \ldots, a_{m}\right)$, $B=\left(b_{1}, b_{2}, \ldots, b_{n}\right)$, then the cumulative distance $\mathrm{D}$ of $\mathrm{A}$ and $\mathrm{B}$ is formula (1):

$$
\begin{aligned}
& D\left(A_{i}, B_{j}\right)=\delta\left(a_{i}, b_{j}\right)+\min \\
& {\left[D\left(A_{i-1}, B_{j-1}\right), D\left(A_{i-1}, B_{j}\right), D\left(A_{i}, B_{j-1}\right)\right]} \\
& \delta\left(a_{i}, b_{j}\right)=\sum_{k}^{n} \frac{\left|a_{i k}-b_{j k}\right|}{\left|a_{i k}\right|+\left|a_{j k}\right|}
\end{aligned}
$$

Where $\left|a_{i k}\right|$ is the absolute value of the $k$ th feature of the $i$ th time point of sequence A. $D\left(A_{i}, B_{j}\right)$ represents the DTW distance value between the $i$ th time point of sequence $\mathrm{A}$ and the $j$ th time point of sequence $B$.

(3) Judge whether it is the first time to calculate the DTW distance. If yes, go to step (5), if not, (4) is preformed.

(4) Judge whether the sum of squares of the DTW distance has decreased, if yes, execute (5), if not, the current average sequence is the result. 
(5) Update each coordinate of the average sequence to the average of the coordinates that match it in the sequence set. Return (2) to continue iteration.

The input of the DBA is a sequence set, and the output is the average of the sequence. From the calculation process, it can be seen that the essence is to calculate the average sequence with the minimum sum of the squared DTW distance of each sequence in the sequence set. This problem is not the traditional problem of calculating extreme value, so heuristic iterative algorithm is adopted to approximate the true value. Fig. 3 and Fig. 4 show the performance of the DBA algorithm. It can be found from the figure that the features clustered by the DBA algorithm completely fit the overall trend of the original curve. It can be seen that this method is feasible.

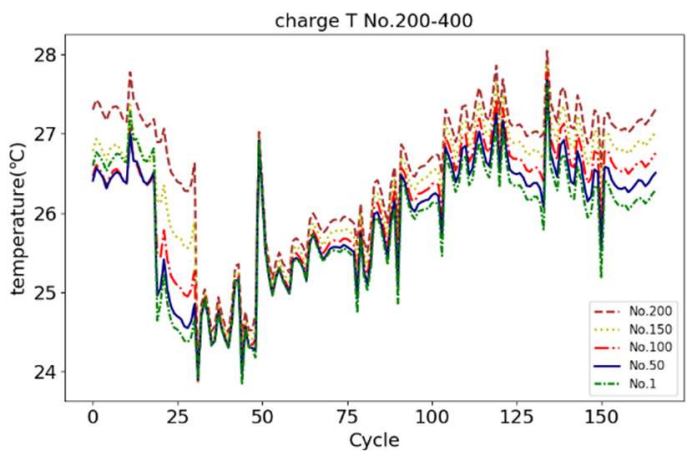

(a)

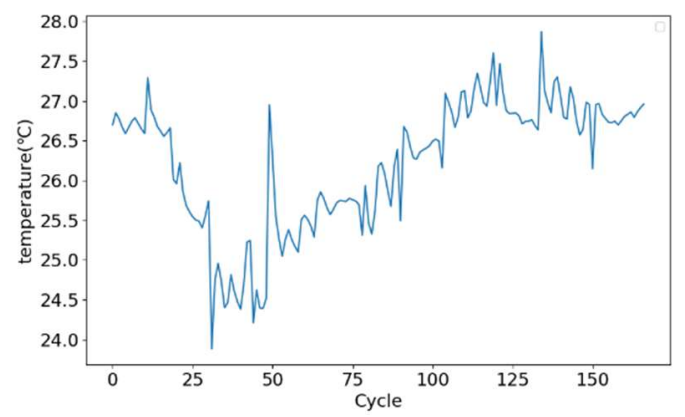

(b)

Fig. 3. (a) Temperature degradation trend curve of B0005 battery NO.200-400. (b) Battery B0005 temperature clustering curve.

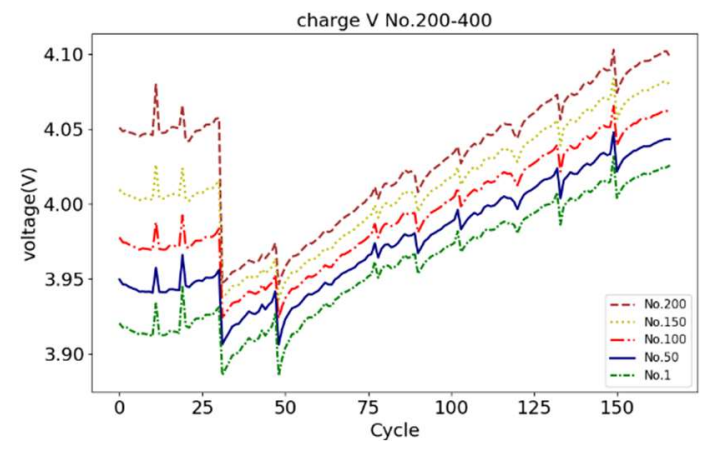

(a)

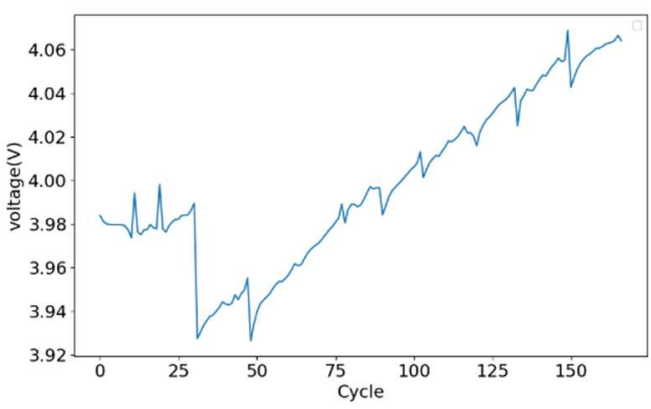

(b)

Fig. 4. (a) Voltage degradation trend curve of B0005 battery NO.200-400. (b) Battery B0005 voltage clustering curve.

\section{Model structure}

Deep convolutional networks have made great breakthroughs in the field of machine vision by virtue of their efficient feature extraction capabilities, and in recent years some convolutional architectures have achieved high accuracy in the field of sequence modeling. TCN network combines many successful applications in the field of convolution at present, confirming that the precision of many sequence modeling tasks is higher than that of RNN [37]. And we have confirmed in the literature [38] that TCN is more effective than the common LSTM and GRU models in predicting battery $\mathrm{SOH}$ in a single-capacity sequence. Therefore, this study improves the TCN model into an AD-TCN model suitable for multi-sequence prediction on the basis of maintaining single-sequence prediction performance. 


\subsection{TCN model}

The TCN model mainly adds dilated convolution and skip connection on the basis of one-dimensional causal convolution. The dilated convolution is proposed to solve the problem of information loss in the process of up-sampling in the image field. The dilated convolution formula for one-dimensional sequences used in this article is as follows:

$\mathrm{F}(i)=\sum_{k=1}^{K} f(i+d \cdot k) \cdot h(k)$

Where $i$ is the $i$ th element in the sequence, $k$ is the size of the filter, $f(i)$ is the input one-dimensional sequence, $\mathrm{F}(i)$ is the output sequence, $h(k)$ is the filter of length $\mathrm{k}$, and $\mathrm{d}$ is dilation factor, for a convolution kernel with size $k^{*} 1$, the size of the dilated filter is $k_{d}^{*} 1$ by inserting a zero value operation.

$k_{d}=k+(k-1) \cdot(d-1)$

Skip connection is a common technique in deep networks, which can improve the speed of the model and ensure the network performance without deterioration. If the model takes $x$ as input and $F(x)$ is the output after a layer of linear transformation and activation, then after the second layer of linear transformation, $F(x)$ is add to the input $\mathrm{x}$ of the previous layer, and then the output is reactivated. The specific formula is as follows:

$o=\operatorname{Activation}(x+F(x))$

In TCN network, the dropout technology, the dilated convolution and the skip connection, plus the activation function constitute a residual module for use. The operating structure of TCN model at $k=2$ is given in Fig. 5. And the paper does not use the ReLU activation function. Considering the phenomenon of 'Dead Neuron' during the experiment, that is, when the input value is negative, the output is always zero, and its first derivative is always zero, resulting in too many neurons will not be updated, so that the prediction result will not change, and the network cannot extract features. Leaky ReLU was finally used as the activation function in the model. By introducing a leakage value in the negative half interval of ReLU, the output had a small slope to the negative value. Since the derivative is not always zero, the phenomenon of neurons dying is reduced, which solves the problem of neurons not learning after the ReLU activation function enters the negative interval.

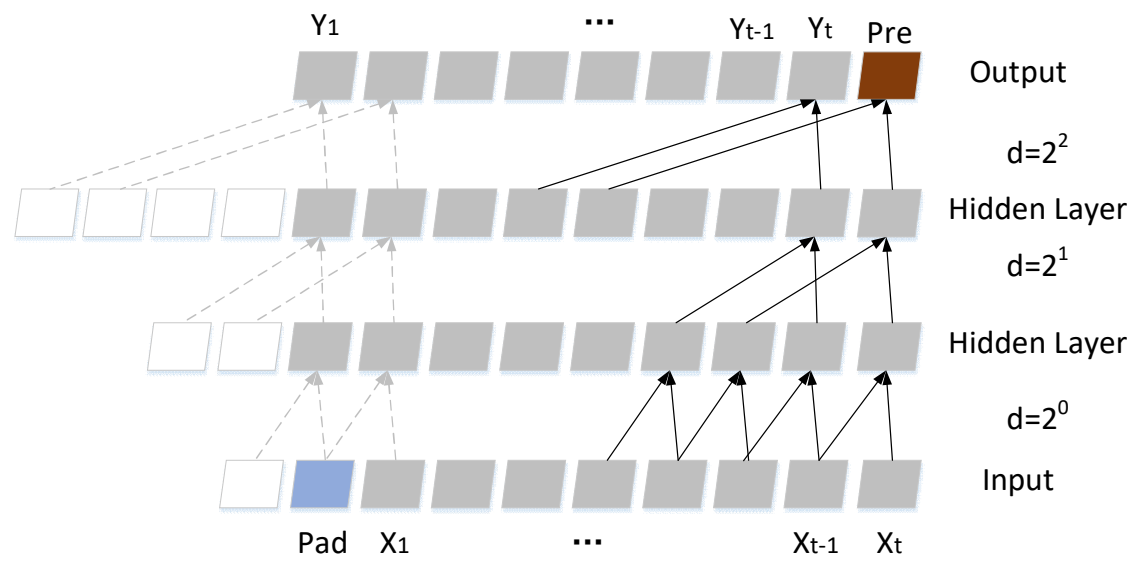

Fig. 5. TCN architecture diagram

To sum up, TCN model has the following advantages and characteristics: 
(1) Based on the causal convolution architecture, combined with the dilated convolution expansion receptive field, so the model itself can consider all historical information, so that the performance of predicting unknown data is more stable. The method to ensure the sequence length is zero padding.

(2) Unlike RNN, its back propagation is independent of the time direction, so exploding gradient problem is hard to occur.

(3) Like CNN, TCN uses the same convolution kernel in each layer, so it can be processed in parallel. Combined with skip connections, a deep network structure can be used.

\subsection{Depthwise separable convolution}

The structure of the TCN model has certain limitations. It is designed for singlesequence modeling tasks, so it can be directly used in $\mathrm{SOH}$ prediction that only considers the capacity sequence. However, considering that the input of the SOH estimation of HFs is twodimensional, this study does not change the advantage of TCN in single-sequence prediction tasks, and makes structural adjustments to TCN through the depthwise separable convolution structure. Depthwise separable convolution is the core means for image feature extraction in the Xception network [39], which is suitable for the same lightweight TCN. Depthwise convolution is used as a multivariate feature extractor, and pointwise convolution reconstructs all extracted features for regression prediction. The specific structure is shown in Fig. 6. In Fig. 6.Suppose that the input is $n \times S, S$ is the length of the sequence, $\mathrm{n}$ is the number of sequences. Unlike conventional convolution, we divide the input into n groups of $S \times 1$, extract the features through the $K \times 1$ convolution kernel to be the output of depthwise, and then pass the features through the convolution kernel of size $1 \times 1$ to convolve ascending dimension output. Compared with the ordinary convolution, the depthwise separable convolution has less parameter amount. When the same convolution kernel is used, the parameter amount of the ordinary convolution is $S \times K \times 1 \times \mathrm{n}$, and the depthwise separable convolution parameter amount is $S \times K \times 1+S \times 1 \times 1 \times$ n. It can be found that the larger the difference between the input and output dimensions $\mathrm{n}$, the fewer the parameters. This also meets the requirements of the TCN network as a lightweight network. Advanced features are further extracted from the input HFs through deep separable convolution, and the features are connected into a one-dimensional vector through pointwise convolution to make regression prediction, so as to fully utilize the advantages of TCN as a lightweight network in single sequence prediction tasks. Therefore, it is feasible to change the single-sequence TCN model to the multi-sequence $\mathrm{SOH}$ estimation model through depthwise separable convolution. 


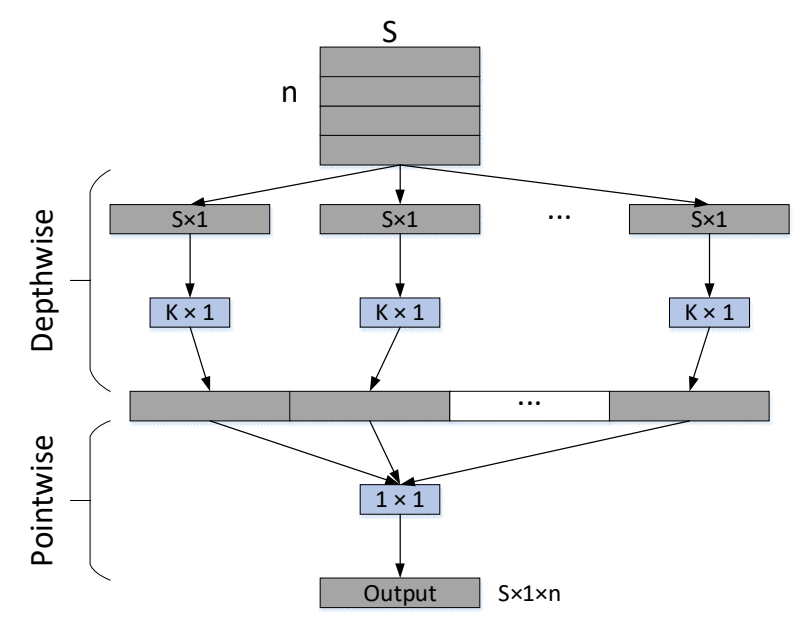

Fig. 6. TCN architecture diagram

\subsection{Convolutional Block Attention Module}

We have integrated the recent convolutional neural network (CNN) improvements in depth and cardinality, in order to improve the model's ability to pay attention to important information, and focus on the factors that really affect the prediction results, rather than just analyzing from the analysis of correlation[40]. The lightweight convolution attention module is integrated into the model. As a method to improve the representation ability of CNN network, it is simple and efficient to improve the learning ability of the model on important features [41]. In this study, it was converted from the field of 3D image processing to $2 \mathrm{D}$ for processing sequence data and embedded into the TCN structure. CBAM is composed of channel attention and spatial attention. It is used to increase the ability of expressing features. Compared with most attention mechanisms that only focus on channels, CBAM is better [42]. Fig. 7 shows the channel attention model after dimensionality reduction. The model first performs average-pooling and max-pooling downsampling on the intermediate feature map in the channel dimension, compresses the global spatial information into a onedimensional vector channel descriptor, and puts it into the multi-layer perceptron (MLP) for adjustment. Finally, the final channel attention feature map $\mathrm{Mc}$ is generated through the sigmoid activation operation. The calculation formula of $\mathrm{Mc}$ is as follows, where

$$
\begin{aligned}
& W_{0} \in R^{C / r \times 1}, \quad W_{1} \in R^{1 \times C / r} 。 \\
& \begin{aligned}
\mathrm{M}_{\mathrm{c}}(F) & =\sigma(\operatorname{MLP}(\operatorname{AvgP\operatorname {Pool}}(F)) \\
& +\operatorname{MLP}(\operatorname{Max} \operatorname{Pool}(F))) \\
& =\sigma\left(W_{1}\left(W_{0}\left(F_{\text {avg }}^{c}\right)\right)+W_{1}\left(W_{0}\left(F_{\max }^{c}\right)\right)\right)
\end{aligned}
\end{aligned}
$$

Perform element wise multiplication of the chanel attention feature map and input feature map to generate the input features required by the spatial attention module. Then in the spatial dimension as shown in Fig. 8, the feature maps processed in the channel dimension are processed through channelbased global-average-pooling and global-maxpooling. Concat the results based on the channel, then reduce the dimension through a convolution operation, and finally output the spatial attention feature Ms through the sigmoid activation function. Ms calculation formula is as follows:

$$
\begin{aligned}
\mathrm{M}_{s}(F) & =\sigma\left(f^{7 \times 7}([\operatorname{AvgPool}(F) ; \operatorname{MaxPool}(F)])\right) \\
& =\sigma\left(f^{7 \times 7}\left(\left[F_{\text {avg }}^{s} ; F_{\max }^{s}\right]\right)\right)
\end{aligned}
$$

By multiplying Ms with the input feature of the module, the final generated feature $\mathrm{F}^{\prime \prime}$ can be obtained. The overall process can be summarized as:

$F^{\prime}=M_{c}(F) \otimes F$

$F^{\prime \prime}=M_{s}\left(F^{\prime}\right) \otimes F^{\prime}$ 


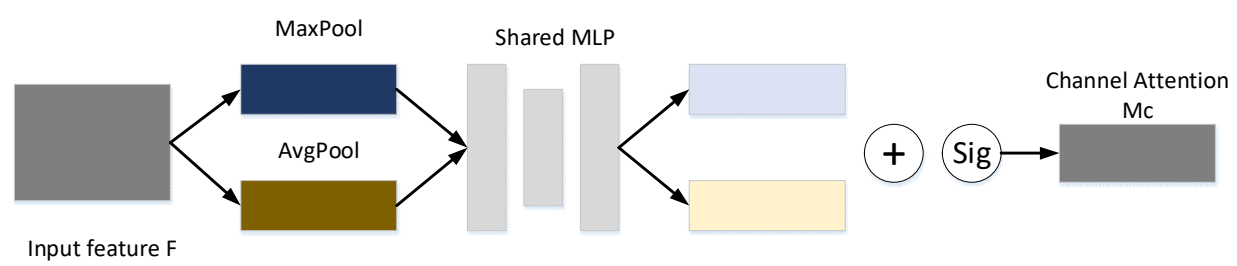

Fig. 7. Channel attention module

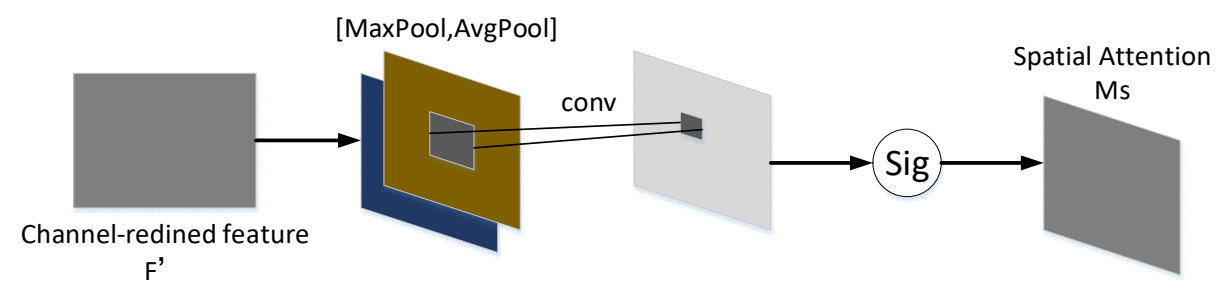

Fig. 8. Spatial attention module

\subsection{AD - TCN model}

The above chapters explain the specific principles of each part of the AD-TCN model. In this chapter, the optimization modules are combined to form the final $\mathrm{SOH}$ estimation model we use. Fig. 9 is the overall $\mathrm{SOH}$ prediction process of this study. The $\mathrm{SOH}$ prediction process of this study is very simple, and although the model used is a deep network, the battery performance data itself is not large, so it is appropriate to use the lightweight ADTCN as the prediction model. In Fig. 9, we carefully analyzed the specific architecture of ad-TCN model and the embedding location of CBAM model for network optimization.
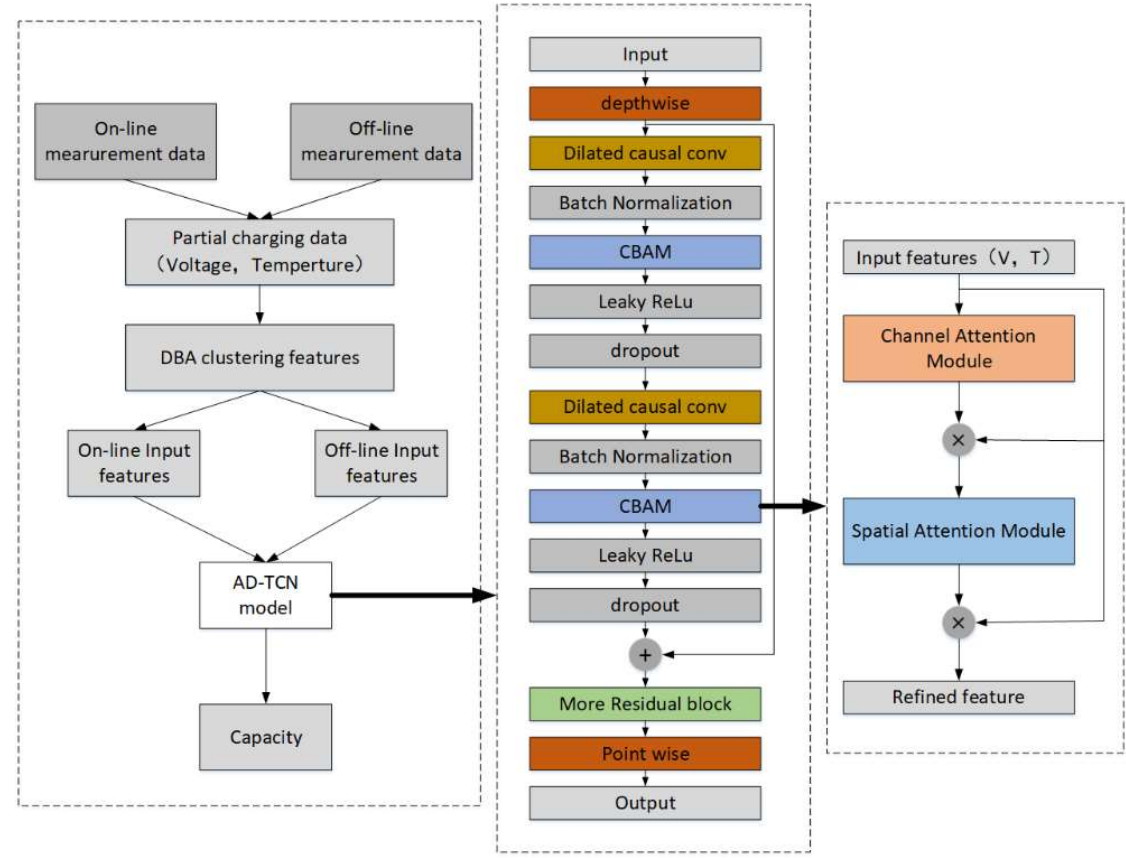

Fig. 9. The overall framework of SOH estimation using AD-TCN model

\section{Analysis of experimental results}

This section analyzes the estimated performance of the model $\mathrm{SOH}$ from two parts: single cell prediction and offline multi-cell prediction. The graph and performance table of $\mathrm{SOH}$ estimation results for different batteries are given by the model, and the results are analyzed. In order to verify the performance of the model in $\mathrm{SOH}$ estimation, root mean square error (RMSE) and mean absolute error (MAE) were used to prove the prediction performance of the AD-TCN model. 


$$
\begin{aligned}
& \text { RMSE }=\sqrt{\frac{1}{n} \sum_{i=1}^{n}\left(y_{i}-\hat{y}_{i}\right)^{2}} \\
& M A E=\frac{1}{n} \sum_{i=1}^{n}\left|\left(y_{i}-\hat{y}_{i}\right)\right|
\end{aligned}
$$

\subsection{Single battery test}

In this study, the performance of the ADTCN model in online prediction is first verified from the $\mathrm{SOH}$ estimation of the single battery, and $50 \%$ of the batteries B0005, B0006, B0007, and B0018 are taken as the training set, and the other $50 \%$ are used as the test set. In Fig. 10, the comparison between the real capacity and the estimated capacity and the error between the model estimation and the test data are given. It can be seen from the figure that the AD-TCN model can well fit the fluctuation of the overall decline trend and local regeneration of the battery. It can also be seen from the error figure that for several batteries used, the overall error can be controlled within $2 \%$. Most of the points with large errors are due to the obvious part of local regeneration phenomenon, and the value of this part has little reference significance for predicting the overall degradation trend of batteries. In addition, the RMSE and MAE values of the single cells are given in Table 2 for the evaluation of model performance.
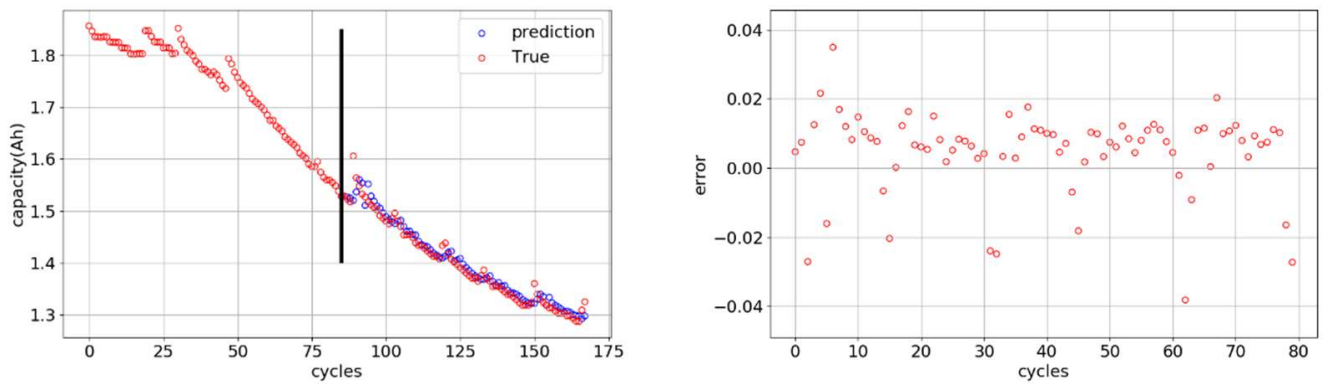

(a) Estimation results and errors of battery B0005
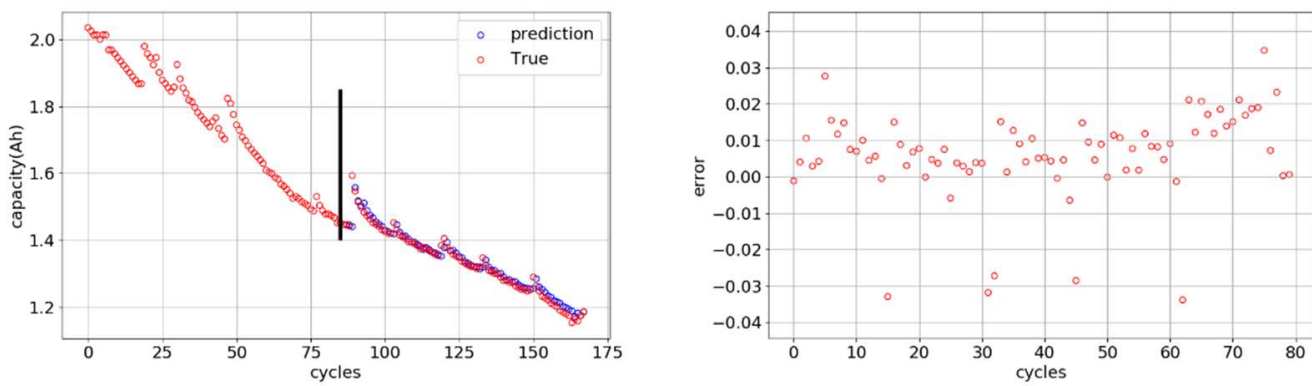

(b) Estimation results and errors of battery B0006
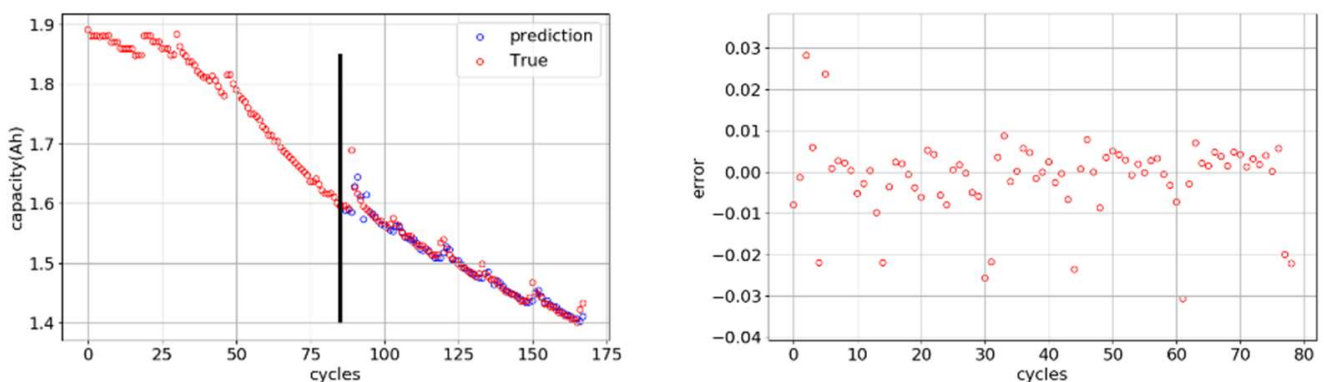

(c) Estimation results and errors of battery B0007 

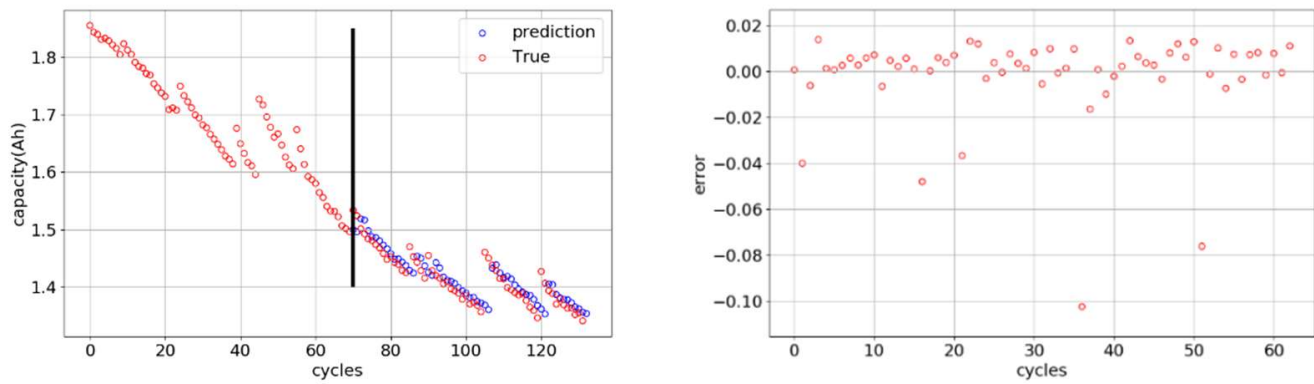

(d) Estimation results and errors of battery B0018

Fig. 10. Single battery experiments

Table 2. Estimation errors of single battery experiments

\begin{tabular}{cccccc}
\hline \hline Method & Battery ID & B0005 & B0006 & B0007 & B0018 \\
\hline \multirow{2}{*}{ LSTM } & RMSE & 0.083 & 0.092 & 0.088 & 0.039 \\
& MAE & 0.072 & 0.068 & 0.080 & 0.033 \\
CNN & RMSE & 0.061 & 0.052 & 0.076 & 0.037 \\
& MAE & 0.054 & 0.041 & 0.072 & 0.031 \\
AD-TCN & RMSE & 0.014 & 0.019 & 0.014 & 0.018 \\
& MAE & 0.011 & 0.011 & 0.006 & 0.011 \\
\hline \hline
\end{tabular}

\subsection{Multiple battery offline estimation}

In this experiment, three batteries in the data set are used as training data, and the other battery data is used as test data to verify the performance of the model used in offline $\mathrm{SOH}$ estimation. It should be noted that this study did not make a prediction for B0006, because the offline training data does not contain all the capacity data of B0006, so the experimental results are not representative. Fig. 11 shows the $\mathrm{SOH}$ estimation results and errors. It can also be seen from the figure that the offline prediction accuracy is relatively low compared to the single cell prediction, but AD-TCN can still control the overall estimation error within $2.5 \%$. From the comparison Table 3 of the two indicators of RMSE and MAE, we can also find that the model has a higher estimation accuracy for batteries B0005 and B0007. Although the local regeneration phenomenon of battery B0018 is more serious, it can also achieve a higher accuracy of the overall degradation trend capture. The accurate estimation of the three batteries verifies the reliability and robustness of the model.
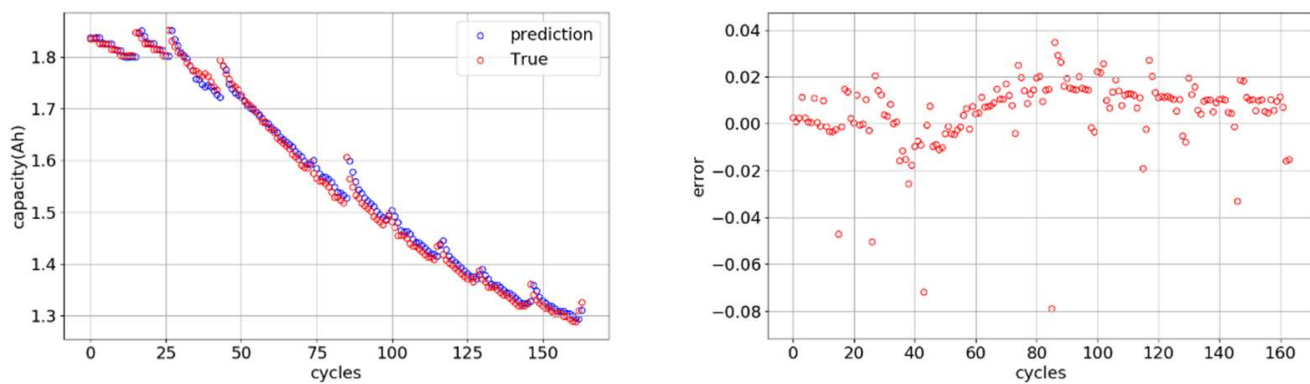

(a) Offline estimation result and error of battery B0005 

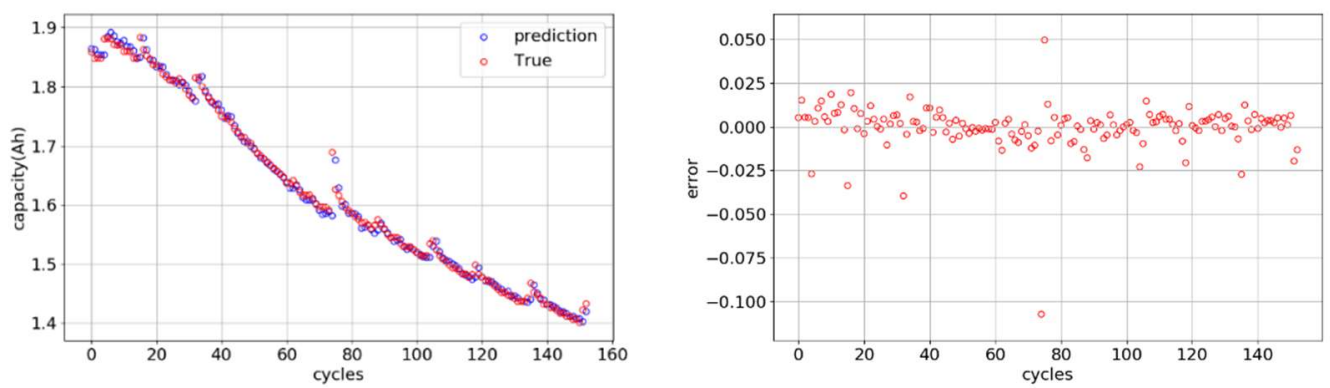

(b) Offline estimation result and error of battery B0007
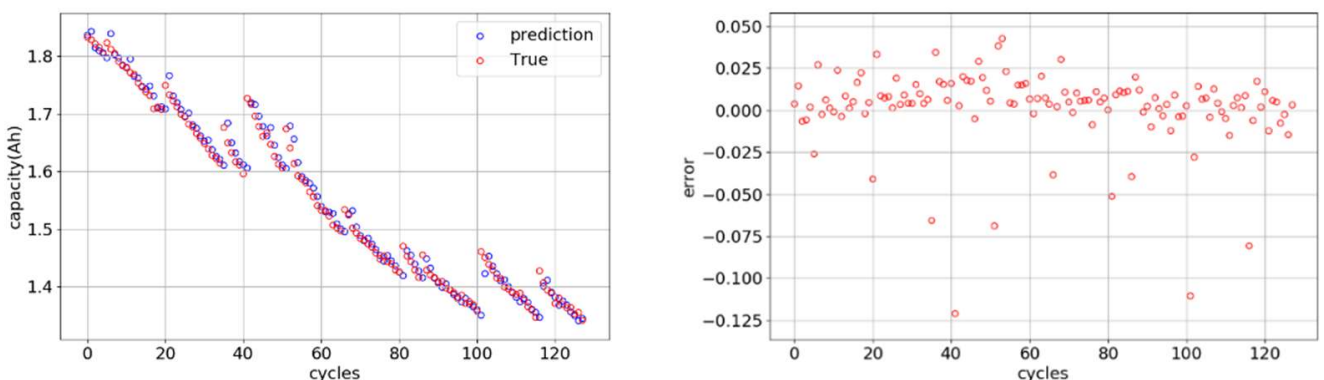

(c) Offline estimation result and error of battery B0018

Fig. 11. Off-line battery experiment

Table 3. Off-line estimation error of multiple batteries

\begin{tabular}{ccccc}
\hline \hline Method & Battery ID & B0005 & B0007 & B0018 \\
\hline \multirow{2}{*}{ LSTM } & RMSE & 0.033 & 0.038 & 0.066 \\
& MAE & 0.031 & 0.035 & 0.054 \\
\multirow{2}{*}{ CNN } & RMSE & 0.045 & 0.051 & 0.070 \\
& MAE & 0.041 & 0.048 & 0.050 \\
AD-TCN & RMSE & 0.014 & 0.012 & 0.021 \\
& MAE & 0.008 & 0.006 & 0.014 \\
\hline \hline
\end{tabular}

\section{Discussion}

In this paper, a new neural network lithium-ion battery health state estimation method based on partial charge degradation characteristics is proposed. From the battery charging process under different health states, it is found that the voltage and temperature have a stable degradation trend, and clustering is performed by the DBA algorithm according to the spatial similarity of the degradation trend, reducing the amount of calculation for directly inputting all data directly. The voltage and temperature sequences after clustering are taken as health characteristic sequences and directly used as the input of the model.

With the development of deep learning in recent years, deep network is rarely applied in the field of battery performance prediction. In this paper, the light TCN network model structure is improved. A new AD-TCN model suitable for multiple prediction is proposed. The structure of the TCN network has been changed through depthwise separable convolution, and depthwise convolution has enabled it to extract features from multiple sequences. Combined with the lightweight convolutional attention mechanism module, compared with the single-channel attention 
mechanism, it can better suppress the secondary features and increase the influence of the main features on the prediction results. Compared with analyzing the correlation degree of the features only, it is more important to improve the influence of the important features on the prediction target. According to the data selected from the NASA dataset, it can be found that the proposed method has better prediction accuracy and is more reliable.

\section{References}

[1] Hannan M A, Lipu M S H, Hussain A, et al. A review of lithium-ion battery state of charge estimation and management system in electric vehicle applications: Challenges and recommendations[J]. Renewable and Sustainable Energy Reviews, 2017, 78: 834-854.

[2] Li Y, Liu K, Foley A M, et al. Data-driven health estimation and lifetime prediction of lithium-ion batteries: A review $[\mathrm{J}]$. Renewable and Sustainable Energy Reviews, 2019, 113: 109254.

[3] Birkl, Christoph R., et al. "Degradation diagnostics for lithium ion cells." Journal of Power Sources 341 (2017): 373-386.

[4] Telmoudi A J, Soltani M, Ben Belgacem Y, et al. Modeling and state of health estimation of nickel-metal hydride battery using an EPSO-based fuzzy cregression model[J]. Soft Computing, 2020, 24(10): 7265-7279

[5] Messing M, Shoa T, Habibi S. Estimating battery state of health using electrochemical impedance spectroscopy and the relaxation effect[J]. Journal of Energy Storage, 2021, 43: 103210.

[6] Cacciato, Mario, et al. "Real-time model-based estimation of $\mathrm{SOC}$ and $\mathrm{SOH}$ for energy storage systems." IEEE Transactions on Power Electronics 32.1 (2016): 794-803

[7] Andre D, Nuhic A, Soczka-Guth T, et al. Comparative study of a structured neural network and an extended Kalman filter for state of health determination of lithium-ion batteries in hybrid electricvehicles[J]. Engineering Applications of Artificial Intelligence, 2013, 26(3): 951-961.
[8] Tang, Xiaopeng, et al. "Aging trajectory prediction for lithium-ion batteries via model migration and Bayesian Monte Carlo method." Applied Energy 254 (2019): 113591.

[9] Fink O, Wang Q, Svensen M, et al. Potential, challenges and future directions for deep learning in prognostics and health management applications[J]. Engineering Applications of Artificial Intelligence, 2020, 92: 103678

[10] Li X , Wang Z , Zhang L , et al. State-of-health estimation for Li-ion batteries by combing the incremental capacity analysis method with grey relational analysis[J]. Journal of Power Sources, 2019, 410-411:106-114.

[11] Zhang H, Miao Q, Zhang X, et al. An improved unscented particle filter approach for lithium-ion battery remaining useful life prediction[J]. Microelectronics Reliability, 2018, 81: 288-298.

[12] Yu, Jianbo. State of Health Prediction of Lithium-Ion Batteries: Multiscale Logic Regression and Gaussian Process Regression Ensemble[J]. Reliability Engineering \& System Safety, 2018:S095183201730652X

[13] Xiao F, Li C, Fan Y, et al. State of charge estimation for lithium-ion battery based on Gaussian process regression with deep recurrent kernel[J]. International Journal of Electrical Power \& Energy Systems, 2021, 124: 106369

[14] Tang T, Yuan H. A hybrid approach based on decomposition algorithm and neural network for remaining useful life prediction of lithium-ion battery[J]. Reliability Engineering \& System Safety, 2021: 108082.

[15] Echevarria Y, Blanco C, Sanchez L. Learning humanunderstandable models for the health assessment of Liion batteries via multi-objective genetic programming $[\mathrm{J}]$. Engineering Applications of Artificial Intelligence, 2019, 86: 1-10.

[16] Patil, Meru A, et al. "A novel multistage Support Vector Machine based approach for Li ion battery remaining useful life estimation." Applied Energy 159.DEC.1(2015):285-297. 
[17] Li, Yuanyuan, et al. "Lithium-ion battery state of health monitoring based on ensemble learning." IEEE Access 7 (2019): 8754-8762.

[18] Yang D, Zhang X, Pan R, et al. A novel Gaussian process regression model for state-of-health estimation of lithium-ion battery using charging curve[J]. Journal of Power Sources, 2018, 384: 387-395.

[19] Guo P , Cheng Z , Yang L . A data-driven remaining capacity estimation approach for lithium-ion batteries based on charging health feature extraction[J]. Journal of Power Sources, 2019, 412:442-450.

[20] Xiaopeng T, Changfu Z, Ke Y, et al. A fast estimation algorithm for lithium-ion battery state of health[J]. Journal of Power Sources, 2018, 396:453-458.

[21] Li X , Wang Z, Zhang L, et al. State-of-health estimation for Li-ion batteries by combing the incremental capacity analysis method with grey relational analysis[J]. Journal of Power Sources, 2019, 410-411:106-114.

[22] Duong P L T, Raghavan N. Heuristic Kalman optimized particle filter for remaining useful life prediction of lithium-ion battery[J]. Microelectronics Reliability, 2018, 81: 232-243.

[23] Benkedjouh T, Medjaher K, Zerhouni N, et al. Remaining useful life estimation based on nonlinear feature reduction and support vector regression $[\mathrm{J}]$. Engineering Applications of Artificial Intelligence, 2013, 26(7): 1751-1760.

[24] Chao M , Xu Z , Zhaopei W, et al. State of health prediction for lithium-ion batteries using multiple-view feature fusion and support vector regression ensemble[J]. International Journal of Machine Learning and Cybernetics, 2018.

[25] Tagade, Piyush, et al. "Deep Gaussian process regression for lithium-ion battery health prognosis and degradation mode diagnosis." Journal of Power Sources 445 (2020): 227281

[26] Richardson, Robert R., Michael A. Osborne, and David A. Howey. "Gaussian process regression for forecasting battery state of health." Journal of Power Sources 357 (2017): 209-219.

[27] Li F, Xu J. A new prognostics method for state of health estimation of lithium-ion batteries based on a mixture of Gaussian process models and particle filter[J]. Microelectronics Reliability, 2015, 55(7): 1035-1045.

[28] Li, Penghua, et al. "State-of-health estimation and remaining useful life prediction for the lithium-ion battery based on a variant long short term memory neural network." Journal of Power Sources 459 (2020): 228069.

[29] Zhang $\mathrm{Y}$, Xiong $\mathrm{R}, \mathrm{He} \mathrm{H}$, et al. Long short-term memory recurrent neural network for remaining useful life prediction of lithium-ion batteries[J]. IEEE Transactions on Vehicular Technology, 2018, 67(7): 5695-5705.

[30] Qu J, Liu F, Ma Y, et al. A Neural-Network-Based Method for RUL Prediction and SOH Monitoring of Lithium-Ion Battery[J]. IEEE Access, 2019, 7: 8717887191 .

[31] Chen J, Jing H, Chang Y, et al. Gated recurrent unit based recurrent neural network for remaining useful life prediction of nonlinear deterioration process[J]. Reliability Engineering \& System Safety, 2019, 185: 372-382.

[32] Xiang S, Qin Y, Zhu C, et al. Long short-term memory neural network with weight amplification and its application into gear remaining useful life prediction[J]. Engineering Applications of Artificial Intelligence, 2020, 91: 103587.

[33] Wen L, Li X, Gao L, et al. A new convolutional neural network-based data-driven fault diagnosis method $[\mathrm{J}]$. IEEE Transactions on Industrial Electronics, 2017, 65(7): 5990-5998.

[34] B. Saha and K. Goebel, "Battery data set," NASA AMES Prognostics Data Repository, 2007.

[35] Petitjean F, Ketterlin A, Gançarski P. A global averaging method for dynamic time warping, with applications to clustering[J]. Pattern Recognition, 2011, 44(3): 678-693.

[36] Sakoe H, Chiba S. Dynamic programming algorithm optimization for spoken word recognition[J]. IEEE transactions on acoustics, speech, and signal processing, 1978, 26(1): 43-49.

[37] Bai S, Kolter J Z, Koltun V. An empirical evaluation of generic convolutional and recurrent networks for sequence modeling $[\mathrm{J}]$. arXiv preprint arXiv:1803.01271, 2018. 
[38] Zhou, Danhua, et al. "State of Health Monitoring and Remaining Useful Life Prediction of Lithium-Ion Batteries Based on Temporal Convolutional Network." IEEE Access 8 (2020): 53307-53320.

[39] Chollet F. Xception: Deep learning with depthwise separable convolutions $[\mathrm{C}] /$ Proceedings of the IEEE conference on computer vision and pattern recognition. 2017: 1251-1258.

[40] Li X, Ding Q, Sun J Q. Remaining useful life estimation in prognostics using deep convolution neural networks[J]. Reliability Engineering \& System Safety, 2018, 172: 1-11.

[41] Woo, Sanghyun, et al. "Cbam: Convolutional block attention module." Proceedings of the European Conference on Computer Vision (ECCV). 2018.

[42] Cui Z, Li Q, Cao Z, et al. Dense attention pyramid networks for multi-scale ship detection in SAR images[J]. IEEE Transactions on Geoscience and Remote Sensing, 2019, 57(11): 8983-8997. 


\section{Supplementary Files}

This is a list of supplementary files associated with this preprint. Click to download.

- declarationofcompetinginterests.docx 